\section{More Tripod Polisher Tips!}

In our May, 1995 issue, we published a tip on using the thpod polisher from Scott D. Walck, Wright-Patterson AFB. As follows are two more tips on the same topic from Scott.

\section{Tripod Polisher Tip \#2}

\section{Preparing the filter paper for the Petri dish:}

I have had two problems with using the filter paper in a Petri dish used for collectung the finished polished sample. The first is that during the various rinsing steps with solvents, the samples would sometimes float around. If I used a sheet of filter paper that fit in the dish, sometimes the sample would drift under the filter paper and the lab would become filled with profane language. The second problem was that if a fibrous filter was used, a fiber from the filter would sometimes "grab" the sample and prevent an epoxy coated grid from picking it up. The following tips are how I solved these problems

1) To solve the pickup problem, I started using a less fibrous filter, Whatman \# 50. This filter is very similar to regular paper in texture. It is a little more difficult to remove the solvents from the dish, but I think that the extra hassle is worth it.

2) To solve the sample floating problem, I combined two solutions. A little more care is needed when rewetting the sample. i slowly add the solvent onto the paper so that the sample will stick to the paper prior to being covered with the solvent. This usually keeps the sample from floating, but not always. To cure the sample from drifting under the filter, I cut the filter paper slightly larger in diameter than the Petri dish. I then center the filter paper on the dish and gently push it into the bottom using a cylindrical jar with a diameter that is about $3 / 4$ of the inside diameter of the dish. I then gently pull the sides of the filter paper up along the sides of the Petri dish. When the sides of the filter paper is about even all the way around the dish, I use the jar to "roll" the sides so that they are perpendicular to the bottom of the filter paper cup. The result resembles a coffee filter with short sides. The edges are sufficiently short so that a cover can be put over the dish.

If the sample does drift close to the edge of the filter paper cup, I can gently lift the cup out of the dish when it is dry and very carefully cut the filter so that I can still maneuver a grid over it under the stereomicroscope, otherwise, 1 mount the specimen on the grid with the filter paper still in the Petrı dish. See Tripod Polishing Tip \#3).

\section{Tripod Polisher Tip \#3 Mounting the Sample onto a Grid:}

I have fat fingers and found that a technique suggested by the IBM group for picking up the samples using a glass slide does not work for me very well. The following steps outline the procedure I use to pick up the final polished samples from the filter paper

1) Prepare the epoxy to be used. Epotek-353ND from Epoxy Technology. inc. works very well. Ron Anderson from IBM says that their group uses M-bond 610 without heat. They let it set overnight.

2) Cut a Post-it sheet into a triangle having about a $30^{\circ}$ angle over the sticky edge.

3) Carefully place the point of the Post-it onto the grid you want to use and firmly press it onto the grid so that you now have a handle for the grid. Bend the Post-It up a IIttle to have a better handle. I typically use $2 \times 1$ slotted grids in either copper, nickel, or beryllium.

4) Carefully paint approximately half the grid with epoxy Use the filter paper that the sample is setting on to blot excess glue.

5) Under the stereomicroscope touch the grid to the sample and pick it up

6) Place the sample on a Teflon block setting on a hot plate with the sample side up and cure the epoxy. (Or wse the IBM technique with M-bond 610.)

I have also used tabbed slotted grids instead of the Post-it paper with good success. Tabbed $2 \times 1$ slotted grids are available from Electron Microscopy Sciences. I have used the copper and nickel. My experience with these grids are that the copper grids work very well, but when you try to bend the tabs on the nickel grids, the tabs break off. A disadvantage to using the tabbed grids is that you must bend the tabs back in the other direction in order to put the sample on the Teflon block with the sample side up to cure the epoxy. This requires additional, precarious handling of the sample before the epoxy is set. I cut the tabs off after the epoxy has cured.

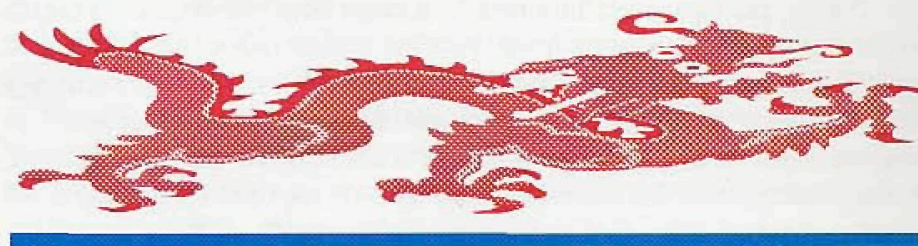

\section{Two For The Price Of One}

\section{Stephen W. Carmichael, Mayo Clinic}

Wouldn't it be great if you could combine the advantages of confocal laser scanning microscopy (CLSM) with the resolving power of atomic force microscopy (AFM)? It almost sounds too good to be true, but you guessed it it's been done! Paul Hillner, Deron Walters, Ratnesh Lal, Helen Hansma, and Paul Hansma of the Department of Physics, University of Californa, Santa Barbara did it in a new and exciting manner

Microscopists are keenly aware that any technique used for preparing and imaging specimens has its own set of problems, particularly the possibility of introducing misleading artifacts. It has long been a practice to confirm the validity of images obtained with a new microscopic technique by comparing it with more established techniques. The combination of the very new AFM with the more established CLSM allows for the comparison of images of the same specimen. Hillner et al. also point out that their combined microscope overcomes certain limitations in the "field of view" because the specimen is scanned past both the AFM cantilever and the area viewed by the objective in the confocal microscope.

The key to the combined microscope is that both the top and bottom of the specimen are accessible for analysis. An objective lens is placed below the specimen, an AFM stylus and cantilever are placed on the upper surface The sample is supported from the side by a piezo tube scanner, angled so that the center of the scan curvature is directly above the microscope objective The really new feature of this combination of microscopes is that a single piezo translator is used to produce the scanning motion for both microscupes The geometric arrangement that aligned the specimen in both microscopes allowed Hilner et al. to scan a Langmuir-Blodgett film of 10,12pentacosadiynoic acid (PCA) up to $300 \mu \mathrm{m}$ on a side, with the AFM and CLSM images in registration. The only limitation to the scan range is the scanning hardware. The scanning rates had to be measured in lines per second, rather than frames per second. This is typical for AFM, but is slow compared to conventional CLSM. Of course it only makes sense that the mode that is slowest for acquiring information be the time-limiting step. While this may be considered a disadvantage of the combined microscope, it surely is offset by the advantages of direct image-to-image comparison. The images of PCA offered in their article demonstrated that small cracks and bumps on the surface could be resolved by AFM and the CLSM yielded other information about the optical properties of the film, albeit at lower resolution

This combined instrument offers the potential of being much more than a novelty. It provides the capability of scanning relatively large areas and providing complimentary information about the specimen. The images are in direct registration, facilitating interpretation of the images. And another advantage is that both images are collected with the same acquisition system, utilizing a single piezo translator with a single set of control and display electronics, thereby reducing cost. This may not be exactly two for the price of one, but it's close!

1. The author gratefully acknowledges Deron A. Walters of the Department of Physics, University of California, Santa Barbara, for reviewing this article. 2 Hillner, P.E., D.A. Walters, R. Lal, H.G. Hansma, and P.K. Hansma, Combined atomic force and confocal laser scanning microscope, Journal of the Microscopy Society of America 1:127-130, 1995. 